\title{
A VIRTUAL REALITY SIMULATOR FOR ACTIVE STEREO VISION SYSTEMS
}

\author{
Manuela Chessa, Fabio Solari, Silvio P. Sabatini \\ Department of Biophysical and Electronic Engineering, University of Genoa - Via all'Opera Pia 11/A - 16145 Genova-ITALY \\ manuela.chessa@unige.it,fabio.solari@unige.it, silvio.sabatini@unige.it
}

Keywords: Binocular vision. Active fixation. Virtual reality. Ground truth data.

\begin{abstract}
The virtual reality is a powerful tool to simulate the behavior of the physical systems. The visual system of a robot and its interplay with the 3D environment can be modeled and simulated through the geometrical relationships between the virtual stereo cameras and the virtual 3D world. The novelty of our approach is related to the use of the virtual reality as a tool to simulate the behavior of active vision systems. In the standard way, the virtual reality is used for the perceptual rendering of the visual information exploitable by a human user. In the proposed approach, a virtual world is rendered to simulate the actual projections on the cameras of a robotic system, thus the mechanisms of the active vision are quantitatively validated by using the available ground truth data.
\end{abstract}

\section{INTRODUCTION}

In 3D computer vision (Trucco and Verri, 1998) and in particular for the stereoscopic vision, it is important to assess quantitatively the progress in the field, but too often the researchers reported only qualitative results on the performance of their algorithms due to the lack of calibrated stereo image databases. To overcome this problem, in the literature we can find works that provide test beds for a quantitative evaluation of the stereo algorithms. Towards this end, the calibrated data sets (Scharstein and Pal, 2007) have to provide both the stereo images and the ground truth disparity map. The left and right intensity patterns observed by the two cameras result related by the binocular disparity map that varies as a function of the spatial position and of the geometry of the vision system. A different approach is to generate stereo image pairs by using a database of range images collected with a laser rangefinder, e.g. (Huang et al., 2000). In this case, we have to compute the stereo projections to obtain the stereo images. In general, the major drawback of the calibrated data sets is the lack of interactivity: it is not possible to change the scene and the camera point of view. The camera position can be slightly modified by using the laser range-finder data, but this move- ment produces undesired occlusions.

The interaction between the visual scene and the vision system is the main characteristic of an active vision system, e.g. a robot system. The paradigm of the active vision was introduced in order to overcome the efficiency and stability caveats of conventional computer vision systems (Aloimonos et al., 1988). A common principle of this paradigm is the behaviordependent processing of visual data by shifting the fixation point on different targets (active foveation) for attentive visual scrutiny. Selective attention and foveation imply the ability to control the mechanical and optical degrees of freedom during image acquisition process (Dankers and Zelinsky, 2004). In such systems the camera movements bring the object of interest in the center of the image pair (by performing camera rotations), and these vergence movements generate both horizontal and vertical disparity (Theimer and Mallot, 1994; Read and Cumming, 2006). These effects can be observed in Fig 1 that shows the real-world images gathered by a binocular robotic head, that is fixating a specific point in the scene through vergence movements.

The aim of this work is to provide a virtual reality tool that implements the requirements imposed by an active vision system and allows the changing of the 


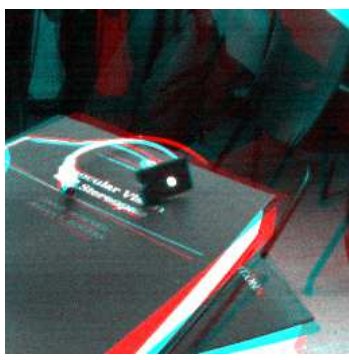

(a)

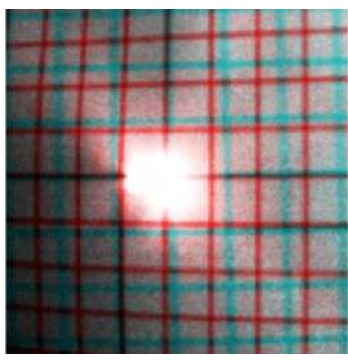

(b)
Figure 1: Binocular snapshots obtained by a real-world active vision system. The two cameras are fixating different objects in the scene (a) the LED on the book and (b) the center of a fronto-parallel grid drawn on a sheet of paper. The anaglyphs are obtained with the left image on the red channel and the right image on the green and blue channels. The interocular distance is $8 \mathrm{~cm}$ and the camera resolution is the standard VGA $640 \times 480$ pixels with a focal length of $6 \mathrm{~mm}$. The distance between the cameras and the objects is between $50 \mathrm{~cm}$ and $90 \mathrm{~cm}$. It is worth noting that both horizontal and vertical disparities are present.

geometry of the virtual stereo cameras as a function of the visual input to the active system. Such a tool, exploiting the ground truth available from the virtual world and the related projected stereo images, provides a way to validate the behavior of an active vision system in a controlled and realistic scenario. The paper is organized as follows: in Section 2 we describe the state of art of the robotic simulators. In Section 3 we briefly introduce the two main methods of setting up virtual cameras and rendering stereo image pairs. In Section 4 we describe the proposed method, the implementation of the active vision behavior and the technique for the ground truth generation. Finally in Section 5 we present the results.

\section{RELATED WORKS}

Recent works on robot simulators address the problem of endowing the systems with visual capabilities (e.g. www. cyberbotics.com), even if the stereo vision is often intended for future developments, e.g. (Jørgensen and Petersen, 2008; Awaad et al., 2008). Other robot simulators in the literature have a binocular vision system. In (Okada et al., 2002) the authors described a simulator of an humanoid robot with 18 degree of freedom (DOF), but only 2 DOF are for the binocular head, thus producing a parallel axis stereo vision. Similarly in (Ulusoy et al., 2004) a virtual robot with an active stereo vision system is presented, but the authors state that they work on stereo image pairs where parallel cameras are used. A virtual hu- man with an active stereo vision system is described in (Rabie and Terzopoulos, 2000), where the two eyes can fixate a target in a scene by computing the stereo disparities between the left and the right foveal images.

Our aim is to simulate an active vision system rather then the whole aspects of a robot acting in an environment (e.g. navigation and mechanical movements of the robot itself). In particular, we aim to precisely simulate the vergence movements of the two cameras in order to provide the stereo views and the related ground truth data (horizontal and vertical disparities and binocular optic flow). Thus, our virtual system can be used for two different purposes: (a) to produce visual behaviors, in a closed loop with a control strategy of the vergence movements guided by a vision-based information; (b) to obtain stereo sequences with related ground truth, to quantitatively assess the performances of computer vision algorithms.

\section{THE COMPUTATION OF THE STEREO IMAGE PAIR}

In the literature the main methods to render stereo image pairs are (Bourke and Morse, 2007): (1) the offaxis technique, usually used to create a perception of depth for a human observer and (2) the toe-in technique that can simulate the actual intensity patterns impinging on the cameras of a robotic head.

In the off-axis technique, the stereo images are generated by projecting the objects in the scene onto the display plane for each camera; such projection plane has the same position and orientation for both camera projections. The model of the virtual setup is shown in Fig 2: F represents the location of the virtual point perceived when looking at the stereo pair composed by $\mathbf{F}^{L}$ and $\mathbf{F}^{R}$. This is the correct way to create stereo pairs that are displayed on stereoscopic devices for human observers. This technique introduces no vertical disparity, thus it does not cause discomfort for the users.

Since our aim is to simulate the actual images acquired by the cameras of a verging pan-tilt robotic head, the correct way to create the stereo pairs is the toe-in method: each camera is pointed at a single focal point (the fixation point) through a proper rotation. The geometrical sketch of the optical setup of an active stereo system and of the related toe-in technique is shown in Fig 3. The relation between the 3D world coordinates $\mathbf{X}=(X, Y, Z)$ and the homogeneous image coordinates $\mathbf{x}^{L}=\left(x^{L}, y^{L}, 1\right)$ and $\mathbf{x}^{R}=\left(x^{R}, y^{R}, 1\right)$ for the toe-in technique is described by a general per- 


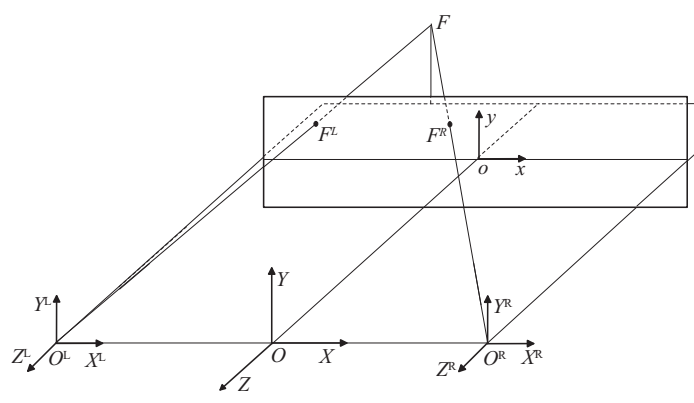

Figure 2: Geometrical sketch of the off-axis technique. The left and right camera frames: $\left(X^{L}, Y^{L}, Z^{L}\right)$ and $\left(X^{R}, Y^{R}, Z^{R}\right)$. The image plane $(x, o, y)$ and the focal length Oo. The image points $\mathbf{F}^{L}$ and $\mathbf{F}^{R}$ are the stereo projection of the virtual point $\mathbf{F}$. The baseline $b$ is denoted by $O^{L} O^{R}$.

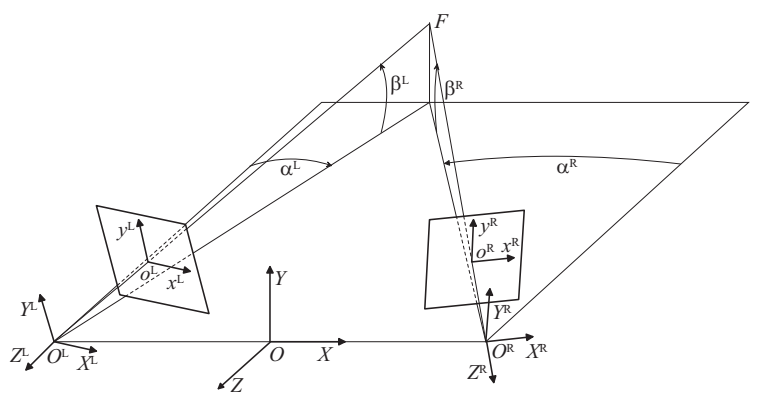

Figure 3: Geometrical sketch of the toe-in technique. The left and right camera frames: $\left(X^{L}, Y^{L}, Z^{L}\right)$ and $\left(X^{R}, Y^{R}, Z^{R}\right)$. The left and right image planes: $\left(x^{L}, o^{L}, y^{L}\right)$ and $\left(x^{R}, o^{R}, y^{R}\right)$. The left and right focal lengths: $O^{L} o^{L}=$ $O^{R} O^{R}$, named $f_{0}$. The camera optical axes $O^{L} F$ and $O^{R} F$ are adjusted to the fixation point $\mathbf{F}$. The baseline $b$ is denoted by $O^{L} O^{R}$, the slant angles by $\alpha^{L}$ and $\alpha^{R}$, and the tilt angles by $\beta^{L}$ and $\beta^{R}$.

spective projection model. A generic point $\mathbf{X}$ in the world coordinates is mapped onto image plane points $\mathbf{x}^{L}$ and $\mathbf{x}^{R}$ on the left and right cameras, respectively. It is worth noting that the fixation point $\mathbf{F}$ in Fig 3 is projected onto the origins of the left and right image planes, since the vergence movement makes the optical axes of the two cameras to intersect in $\mathbf{F}$. For identical left and right focal lengths $f_{0}$, the left image coordinates are (Volpel and Theimer, 1995):

$$
\begin{aligned}
x^{L} & =f_{0} \frac{X_{+} \cos \alpha^{L}+Z \sin \alpha^{L}}{X_{+} \sin \alpha^{L} \cos \beta^{L}-Y \sin \beta^{L}-Z \cos \alpha^{L} \cos \beta^{L}} \\
y^{L} & =f_{0} \frac{X_{+} \sin \alpha^{L} \sin \beta^{L}+Y \cos \beta^{L}-Z \cos \alpha^{L} \sin \beta^{L}}{X_{+} \sin \alpha^{L} \cos \beta^{L}-Y \sin \beta^{L}-Z \cos \alpha^{L} \cos \beta^{L}}
\end{aligned}
$$

where $X_{+}=X+b / 2$. Similarly, the right image coordinates are obtained by substituting in the previous equations $\alpha^{R}, \beta^{R}$ and $X_{-}=X-b / 2$. We can define the horizontal disparity $d_{x}=x^{R}-x^{L}$ and the vertical disparity $d_{y}=y^{R}-y^{L}$, that establish the relationships between a world point $\mathbf{X}$ and its associated disparity vector $\mathbf{d}=\left(d_{x}, d_{y}\right)$. The disparity patterns produced by the off-axis and toe-in techniques are shown in Fig 4a and Fig 4b, respectively.

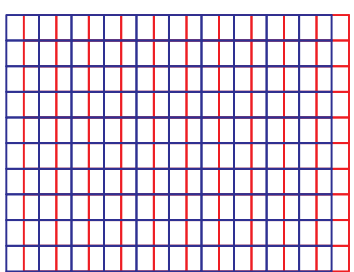

(a)

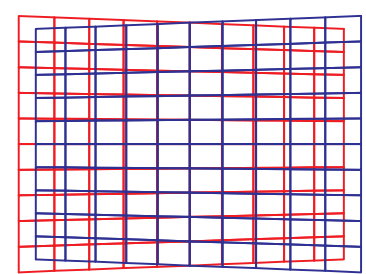

(b)
Figure 4: The projections of a fronto-parallel square onto the image planes, drawn in red for the left image and blue for the right. The texture applied to the square is a regular grid. (a) The projection obtained with the off-axis technique: only horizontal disparity is introduced. (b) The projection obtained with the toe-in technique: both vertical and horizontal disparities are introduced. The projected patterns resemble the ones obtained in real-world situations (see Fig. 1b).

\section{IMPLEMENTATION}

The virtual reality tool we propose in this paper is based on a $\mathrm{C}++$ / OpenGL architecture and on the Coin3D graphic toolkit (www. coin3D.org). Coin3D is built on OpenGL and uses scene graph data structures to render $3 \mathrm{D}$ graphics in real time. Both OpenGL and Coin3D code co-exist in our application.

\subsection{Stereo implementation}

To implement the stereo geometry described in the Section 3 we modified the SoCamera node in Coin3D distribution. The SoCamera class is the abstract base class for camera definition nodes and it can be used to obtain a stereoscopic visualization of the scene. The stereoscopic technique usually implemented is the off-axis technique, described in Section 3. Our aim is to add the toe-in technique, to generate stereo pairs like in a pan-tilt robotic head.

Accordingly, we introduced the possibility of pointing the left and the right views at a single focal point, keeping fixed and symmetric the two view volumes and rotating them. To obtain the left and the right views both fixating a point $\mathbf{F}$, a symmetric view volume is created, centered in the position $\mathbf{O}=$ $(X, Y, Z)$ (see Fig. 3). The skewed frustum (necessary to obtain the off-axis stereo technique) is no longer 
necessary. The view volume is then translated to the positions $\mathbf{O}^{L}=\left(X^{L}, Y^{L}, Z^{L}\right)$ and $\mathbf{O}^{R}=\left(X^{R}, Y^{R}, Z^{R}\right)$ in order to obtain the stereo separation $b$. The translation for the left and the right view volume can be obtained by applying the following translation matrix:

$$
\mathbf{T}^{L / R}=\left[\begin{array}{cccc}
1 & 0 & 0 & \pm \frac{b}{2} \\
0 & 1 & 0 & 0 \\
0 & 0 & 1 & 0 \\
0 & 0 & 0 & 1
\end{array}\right]
$$

Then the azimuthal rotation $\left(\alpha^{L}\right.$ and $\left.\alpha^{R}\right)$ and the elevation $\left(\beta^{L}\right.$ and $\left.\beta^{R}\right)$ are obtained with the following rotation matrices:

$$
\begin{aligned}
\mathbf{R}_{\alpha}^{L / R}= & {\left[\begin{array}{cccc}
\cos \alpha^{L / R} & 0 & \sin \alpha^{L / R} & 0 \\
0 & 1 & 0 & 0 \\
-\sin \alpha^{L / R} & 0 & \cos \alpha^{L / R} & 0 \\
0 & 0 & 0 & 1
\end{array}\right] } \\
\mathbf{R}_{\beta}^{L / R}= & {\left[\begin{array}{cccc}
1 & 0 & 0 & 0 \\
0 & \cos \beta^{L / R} & -\sin \beta^{L / R} & 0 \\
0 & \sin \beta^{L / R} & \cos \beta^{L / R} & 0 \\
0 & 0 & 0 & 1
\end{array}\right] }
\end{aligned}
$$

The complete roto-translation of the viewvolumes is:

$$
\left[\begin{array}{c}
\mathbf{O}^{L / R} \\
1
\end{array}\right]=\mathbf{R}_{\beta}^{L / R} \mathbf{R}_{\alpha}^{L / R} \mathbf{T}^{L / R}\left[\begin{array}{c}
\mathbf{O} \\
1
\end{array}\right]
$$

Thus, the projection direction is set to the target point $\mathbf{F}$, then the left and the right views project onto two different planes, as it can be seen in Fig 3 .

In this way, it is possible to insert a camera in the scene (e.g. a perspective camera), to obtain a stereoscopic representation with convergent axis and to decide the location of the fixation point. This emulates the behavior of a couple of verging pan-tilt cameras.

\subsection{Active vision implementation}

The described tool is active in the sense that the fixation point $\mathbf{F}$ of the stereo cameras varies to explore the scene. We can distinguish two possible scenarios: (1) to use the system to obtain sequences where the fixation points are chosen on the surfaces of the objects in the scene; (2) to use the system in cooperation with an algorithm that implements a vergence/version strategy. In the first case, it is not possible to fixate beside or in front of the objects. In the second case, the vergence/version algorithm gives us an estimate of the fixation point, the system adapts itself looking at this point and the snapshots of the scene are then used as a new input for selecting a new target point.

In this work, we focused on the first issue, and we want the system to fixate points laying on the objects' surfaces. To this end, it is necessary to derive the $3 \mathrm{D}$ coordinates of all the visible surfaces.
This information can be obtained from the z-buffer with the glReadPixels function, from which we obtain the $3 \mathrm{D}$ window coordinates, that are mapped into the object coordinates, through the function gluUnproject, by using the transformations defined by the ModelView matrix, the Projection matrix and the Viewport (Hearn and Baker, 1997; Wrigh et al., 2007).

\subsection{Ground truth data generation}

To compute the ground truth data for the horizontal and vertical disparities of the stereo image pairs, given the projection of a $3 \mathrm{D}$ virtual point in one image plane, we have to look for the correspondent projection in the other image plane. Formally, the two camera reference frames are related by a rigid body transformation described by the rotation matrix $\mathcal{R}$ and the translation $\mathcal{T}$. The left and right projections are related by the same transformation in the following way (Ma et al., 2004):

$$
\lambda^{R} \mathbf{x}^{R}=\mathcal{R} \lambda^{L} \mathbf{x}^{L}+\mathcal{T}
$$

where $\mathbf{x}^{L}$ and $\mathbf{x}^{R}$ are the homogeneous coordinates in the two image planes, and $\lambda^{L}$ and $\lambda^{R}$ are the depth values.

To apply the relationship described by Eq. 5 we first read the $\mathrm{z}$-buffer $(w)$ of the two stereo views through the glReadPixels function, then we obtain the depth values with respect to the reference frames of the two cameras in the following way:

$$
\lambda^{L / R}=\frac{f n}{w^{L / R}(f-n)-f}
$$

where $f$ and $n$ represent the values of the far and the near planes of the virtual camera. Starting from the image coordinate $\mathbf{x}^{L}$ of the left image and the depth values $\lambda^{L / R}$ obtained by Eq. 6 , we obtain the image coordinate $\mathbf{x}^{R}$ of the right view by combining the rototranslation described in Eq. 4 and Eq. 5 in the following way:

$$
\lambda^{R} \mathbf{x}^{R}=\mathbf{R}^{R} \mathbf{T}^{R}\left(\mathbf{T}^{L}\right)^{-1}\left(\mathbf{R}^{L}\right)^{-1} \lambda^{L} \mathbf{x}^{L}
$$

where $\mathbf{R}^{L / R}=\mathbf{R}_{\beta}^{L / R} \mathbf{R}_{\alpha}^{L / R}$. Finally the horizontal disparity $d_{x}=x^{R}-x^{L}$ and the vertical disparity $d_{y}=$ $y^{R}-y^{L}$ are computed.

\section{RESULTS}

The described tool produces couples of stereo images, from a pair of stereoscopic vergent cameras in a scene where the $3 \mathrm{D}$ coordinates of the objects and 
their 2D projections are known and controlled. The fixation point of the two cameras is set by using the actual depth values referred to the cyclopic position, moreover the ground truth disparities between the two views are computed.

Figure 5c-d shows the typical horizontal and vertical disparity patterns that emerge when two cameras are fixating the center of a fronto-parallel plane. It is worth noting that these patterns are similar both to the geometrically obtained projections (see Fig. 4b) and to the disparities present in the images acquired by a real-world active vision system (see Fig. 1b). Since our aim is to obtain a simulator that behaves like a robotic binocular head in a real-world environment, it is necessary to create quite complex scenarios. In particular, it is necessary to have different objects at different depths, with "realistic" textures, in order to create benchmark sequences of appropriate complexity. In Fig 6 we present some examples for a virtual environment representing a typical indoor situation (an office). The simulator aims to mimic the behavior of an active system with human-like features acting in the peripersonal space, thus the interocular distance between the two cameras is set to $6.5 \mathrm{~cm}$ and the distance between the cameras and the objects ranges between 80 and $90 \mathrm{~cm}$. The different fixation points have been chosen randomly, by using the depth map of Fig 6b, where dark gray values correspond to far objects, while light gray values correspond to near objects. The aim of this test was to create a set of images gathered by different fixation points, thus simulating an active exploration of the scene. The results of this exploration are shown in Fig 6c-f. It is worth noting that in the proximity of the fixation point the disparity between the left and the right projections is zero, while getting far from the fixation point both horizontal and vertical disparities emerge (Theimer and Mallot, 1994; Read and Cumming, 2006), as it can be seen in the ground truth data of Fig 7.

\section{CONCLUSIONS AND FUTURE WORK}

We have described a virtual reality tool, capable of generating pairs of stereo images like the ones that can be obtained by a verging pan-tilt robotic head and the related ground truth data.

To obtain such a behavior the toe-in stereoscopic technique should be preferred to the off-axis technique. By proper rototranslations of the view volumes, we can create benchmark sequences for vision systems with convergent axis. Moreover, by using the precise $3 \mathrm{D}$ position of the objects these

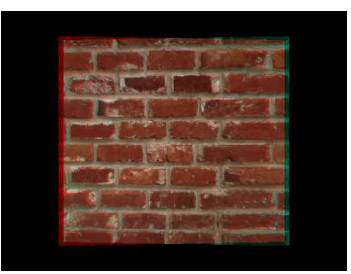

(a)

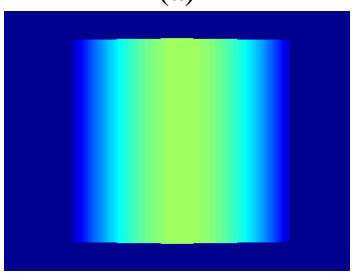

(c)

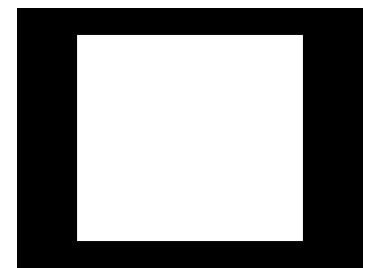

(b)

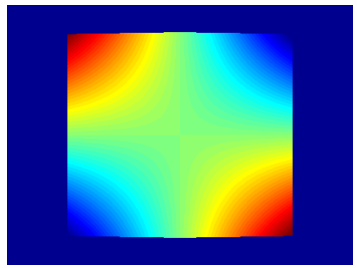

(d)
Figure 5: (a) Anaglyph of a stereo pair obtained from the two virtual cameras fixating the center of a fronto-parallel plane. (b) Depth map referred to the cyclopic position. (c-d) Horizontal and vertical ground truth disparity maps, coded from red (positive values of disparity) to blue (negative values). Zero disparity is coded in green.

vision systems can interact with the scene in a proper way. A data set of stereo image pairs and the related ground truth disparities are available for the Computer Vision community at the web site www.pspc.dibe.unige.it/Research/vr.html.

Since the purpose of this work was not to create a photo-realistic virtual reality tool but to obtain sufficiently complex scenarios for benchmarking an active vision system, we have not directly addressed the problem of improving the photo-realistic quality of the 3D scene, rather we focused on the definition of a realistic model of the interactions between the vision system and the observed scene. The creation of even more complex and photo-realistic scenes will be part of a future work.

Furthermore, we will integrate vergence/version strategies in the system in order to have a really active tool that interacts with the virtual environments. It would also be interesting to modify the standard pan-tilt behavior by including more biologically plausible constraints on the camera movements (Schreiber et al., 2001; Van Rijn and Van den Berg, 1993).

\section{Acknowledgements}

We wish to thank Luca Spallarossa for the helpful comments. This work has been partially supported by EU Projects FP7-ICT 217077 "EYESHOTS" and FP7-ICT 215866 "SEARISE”. 


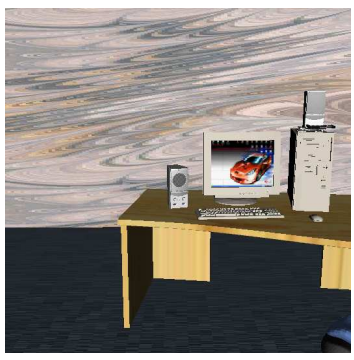

(a)

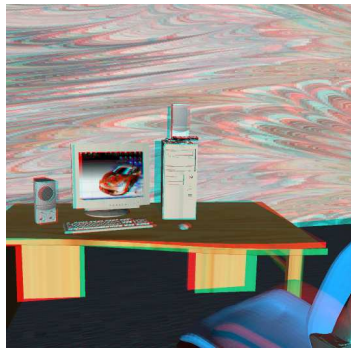

(c)

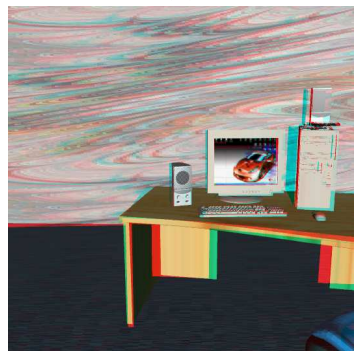

(e)

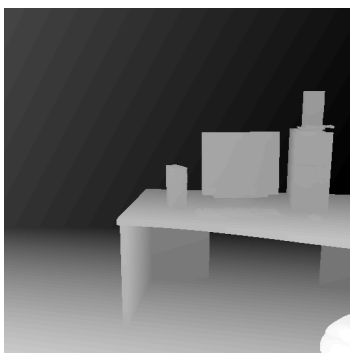

(b)

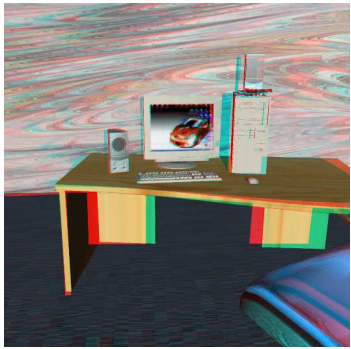

(d)

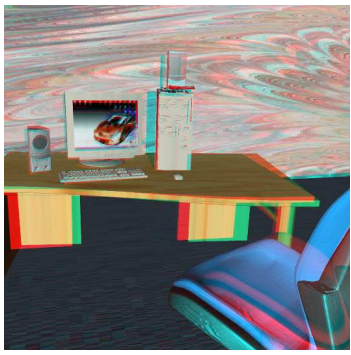

(f)
Figure 6: Snapshots obtained by our simulator of an active vision system. (a) The virtual scenario used for testing the developed tool. (b) Depth map referred to the initial position of the cameras. The stereoscopic cameras are exploring the scene by fixating different objects in the scene: (c) the case, (d) the keyboard, (e) the left speaker and (f) the mouse.

\section{REFERENCES}

Aloimonos, Y., Weiss, I., and Bandyopadhyay, A. (1988). Active vision. Int. J. of Computer Vision, 1:333-356.

Awaad, I., Hartanto, R., León, B., and Plöger, P. (2008). A software system for robotic learning by experimentation. In Workshop on robot simulators (IROSO8).

Bourke, P. and Morse, P. (2007). Stereoscopy: Theory and practice. Workshop at 13th International Conference on Virtual Systems and Multimedia.

Dankers, A. and Zelinsky, A. (2004). Cedar: A real-world vision system: Mechanism, control and visual processing. Machine Vision and Appl., 16(1):47-58.

Hearn, D. and Baker, M. P. (1997). Computer Graphics, C Version. 2nd edition. Prentice Hall.

Huang, J., Lee, A. B., and Mumford, D. (2000). Statistics of range images. In Proc. of the IEEE Conference on Computer Vision and Pattern Recognition.

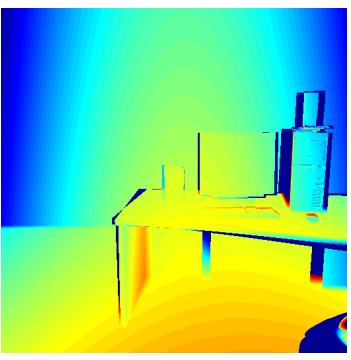

(a)

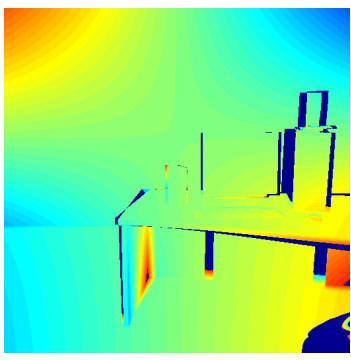

(b)
Figure 7: The horizontal and vertical ground truth disparity maps of the stereo pair of Fig 6e. Occlusions are marked with dark blue.

Jørgensen, J. and Petersen, H. (2008). Usage of simulations to plan stable grasping of unknown objects with a 3-fingered schunk hand. In Workshop on robot simulators (IROSO8).

Ma, Y., Soatto, S., and Kosecka, J.and Sastry, S. (2004). An Invitation to $3 D$ Vision. From Images to Geometric Models. Springer-Verlag.

Okada, K., Kino, Y., and Kanehiro, F. (2002). Rapid development system for humanoid vision-based behaviors with real-virtual common interface. In IEEE/RSJ Int. Conf. on Intelligent Robots and Systems.

Rabie, T. F. and Terzopoulos, D. (2000). Active perception in virtual humans. In Vision Interface 2000 (VI 2000).

Read, J. and Cumming, B. (2006). Does depth perception require vertical disparity detectors? Journal of Vision, 6(12):1323-1355.

Scharstein, D. and Pal, C. (2007). Learning conditional random fields for stereo. In Proc. of the IEEE Conference on Computer Vision and Pattern Recognition.

Schreiber, K. M., Crawford, J. D., Fetter, M., and Tweed, D. B. (2001). The motor side of depth vision. Nature, 410:819-822.

Theimer, W. and Mallot, H. (1994). Phase-based binocular vergence control and depth reconstruction using active vision. CVGIP: Image Understanding, 60(3):343358.

Trucco, E. and Verri, A. (1998). Introductory Techniques for 3-D Computer Vision. Prentice Hall.

Ulusoy, I., Halici, U., and Leblebicioglu, K. (2004). 3d cognitive map construction by active stereo vision in a virtual world. Lecture notes in Computer Science, 3280:400-409.

Van Rijn, L. and Van den Berg, A. (1993). Binocular eye orientation during fixations: Listing's law extended to include eye vergence. Vision Research, 33:691-708.

Volpel, B. and Theimer, W. (1995). Localization uncertainty in area-based stereo algorithms. IEEE Transactions on Systems, Man and Cybernetics, 25(12):1628-1634.

Wrigh, R., Lipchack, B., and Haemel, N. (2007). OpenGL superbible, Fourth Edition, Comprehensive Tutorial and Reference. Addison-Wesley. 